\title{
State Estimation for Fractional-Order Complex Dynamical Networks with Linear Fractional Parametric Uncertainty
}

\author{
Hongjie Li \\ College of Mathematics and Information and Engineering, Jiaxing University, Zhejiang, 314001, China \\ Correspondence should be addressed to Hongjie Li; lhjymlly@163.com
}

Received 30 October 2012; Revised 8 February 2013; Accepted 13 February 2013

Academic Editor: Juan J. Trujillo

Copyright (C) 2013 Hongjie Li. This is an open access article distributed under the Creative Commons Attribution License, which permits unrestricted use, distribution, and reproduction in any medium, provided the original work is properly cited.

\begin{abstract}
This paper deals with state estimation problem for a class of fractional-order complex dynamical networks with parametric uncertainty. The parametric uncertainty is assumed to be of linear fractional form. Firstly, based on the properties of Kronecker product and the stability of fractional-order system, a sufficient condition is derived for robust asymptotic stability of linear fractional-order augmented system. Secondly, state estimation problem is then studied for the same fractional-order complex networks, where the purpose is to design a state estimator to estimate the network state through available output measurement, the existence conditions of designing state estimator are derived using matrix's singular value decomposition and LMI techniques. These conditions are in the form of linear matrix inequalities which can be readily solved by applying the LMI toolbox. Finally, two numerical examples are provided to demonstrate the validity of our approach.
\end{abstract}

\section{Introduction}

Complex networks have become a focus of research and have been paid much attention from various fields of the science of engineering during the past few years, due to many realworld systems can be described by complex networks, such as the World Wide Web, neural networks, social networks, cooperate networks, and food webs, electrical power grids [112]. In particular, stability and synchronization of complex dynamical networks have received great attention and many profound results have been established [13-20].

Owing to the complexity of large-scale networks, state of the node is not often fully available in the network outputs; moreover, in many practical applications, one needs to know the state's information and then use it to achieve certain objectives; therefore, it becomes necessary to estimate state of the key node through available measurements; state estimation problem of complex dynamical networks became an important topic recently [21-28], which employ the stochastic analysis techniques and the properties of Kronecker product to establish delay-dependent criteria that ensure the existence of desired estimator gains. Particularly, [21] investigates state estimation problem for delayed neural networks and derives some delay-independent sufficient conditions for the existence of an estimator. It is noticed that discrete-time networks have a better position to model digital transmitted signals in a dynamical way than continuous-time networks. Recently, state estimation problem for discrete-time networks has received some research interests, such as synchronization and state estimation being investigated in [22] for discretetime complex networks with distributed delays, and an LMI approach is developed to design state estimator. In [25], a novel synchronization problem has been discussed for a class of discrete time-varying stochastic complex networks over a finite horizon, and the bounded $H_{\infty}$ synchronization criteria have been established in terms of a set of recursive linear matrix inequalities. Although, most of the studies focus on synchronization and state estimation of integerorder complex networks, many phenomena in nature cannot be explained in the framework of integer-order dynamics, for example, the synchronized motion of agents in fractional circumstances, such as molecule fluids and porous media; the stress-strain relationship demonstrates noninteger-order dynamics rather than integer-order dynamics [29]. For the fractional-order dynamical systems [30-32], it is very difficult and inconvenient to construct Lyapunov functions because there exist substantial differences between fractional-order differential systems and integer-order differential ones. As a 
way of efficiently solving the robust stability and stabilization problem, linear matrix inequality approach is presented [33-36], which provides sufficient condition and designing method of state feedback controllers for fractional-order systems. As an extension of their application, recently, the collective dynamics analysis of fractional-order complex networks has led to a host of interesting effects [37-39]. In [37], the pinning control problem of fractional-order weighted complex dynamical networks is considered for the first time; some local stability properties of such pinned fractionalorder networks are derived and the valid stability regions are estimated. Based on the stability theory of fractionalorder system, [38] derives the sufficient criteria for outer synchronization by applying the nonlinear control and the bidirectional coupling methods. It is noticed that uncertainty is unavoidable because it is very difficult to obtain an exact mathematical model due to environmental noise. Reference [39] investigates robust synchronization of fractional-order complex dynamical networks with parametric uncertainties, some robust synchronization criteria are derived by applying the nonlinear control, and the above uncertainty is described by the norm bounded uncertainty. Recently, a new type of uncertainty, namely, linear fractional form is considered in [40-42], which can include the norm-bounded uncertainties as a special case. Comparing with norm-bounded uncertainties, the formulation of linear fractional uncertainties can obtain less conservative results. To the best of our knowledge, no result has been reported on state estimation for fractionalorder complex dynamical networks with linear fractional parametric uncertainty, which is very important in both theories and applications and is also a very challenging problem. The main purpose of this paper is to address these shortcomings.

Motivated by the above discussion, this paper focuses on state estimation for fractional-order complex dynamical networks with linear fractional parametric uncertainty. Firstly, based on the properties of Kronecker product and stability of the fractional-order system, a sufficient condition is derived for robust asymptotic stability of linear fractional-order augmented system. Secondly, state estimation problem is then studied for the same fractional-order complex networks; the existence condition and method of designing state estimator are derived by using matrix's singular value decomposition and LMI techniques. These conditions are in the form of linear matrix inequalities, which can be readily solved by applying the LMI toolbox.

The main novelty of this paper can be summarized as follows: (1) state estimation problem for fractional-order complex dynamical networks with parametric uncertainty is considered in this paper, where the parametric uncertainty is assumed to be of linear fractional form. (2) A novel state estimator is constructed, in addition to estimator matrix to be designed; an unknown estimator state matrix is also involved. (3) The existence condition and method of designing state estimator are derived by using matrix's singular value decomposition and LMI techniques.

Notation. The notation used here is fairly standard. $\mathbb{R}^{n}$ denotes the $n$-dimensional Euclidean space, and $\mathbb{R}^{n \times m}$ is the set of real $n \times m$ matrices. The superscript $T$ represents the transpose of matrix (or vector). I denotes the identity matrix of compatible dimensions. In symmetric block matrices, $*$ is used as an ellipsis for terms induced by symmetry. $\operatorname{diag}\{\ldots\}$ stands for a block-diagonal matrix. The notation $X \geq 0(X>$ 0 ) means that $X$ is positive semidefinite (positive definite). $\operatorname{Sym}\{X\}$ denotes the expression $X+X^{T}$. The Kronecker product of an $n \times m$ matrix $X$ and a $p \times q$ matrix $Y$ is defined by an $n p \times m q$ matrix $X \otimes Y=\left(x_{i j} Y\right)_{n p \times m q}$. If they are not explicitly specified, arguments of a function or a matrix will be omitted in the analysis when no confusion can arise.

\section{Problem Formulation and Preliminaries}

Consider the following fractional-order complex dynamical networks consisting of $N$ nodes:

$$
\begin{aligned}
& { }_{C} D_{t_{0}, t}^{\alpha} x_{i}(t)=A x_{i}(t)+\sum_{j=1}^{N} G_{i j} \Gamma x_{j}(t), \\
& y_{i}(t)=C x_{i}(t) \quad(i=1,2, \ldots, N),
\end{aligned}
$$

where $\alpha \in[1,2)$ is the fractional order. $x_{i}(t) \in \mathbb{R}^{n}$ is state vector of the $i$ th node, and $y_{i}(t) \in \mathbb{R}^{m}$ is the output vector, $G=\left(G_{i j}\right)_{N \times N}$ is coupled configuration matrix of the networks with $G_{i j} \geq 0(i \neq j)$, the diagonal elements of coupling configuration matrix are defined as $G_{i i}=-\sum_{j=1, j \neq i} G_{i j}(i=$ $1,2, \ldots, N)$, and $\Gamma \in \mathbb{R}^{n}$ is an inner-coupling matrix. $A$ and $C$ are system matrices with appropriate dimensions. Further, $A=A_{0}+\Delta A(t)$, where $\Delta A(t)$ denotes the time-varying, and linear fractional norm-bounded uncertainty is described by

$$
\Delta A(t)=D_{A} \Delta(t) E_{A},
$$

where $D_{A}$ and $E_{A}$ are two known constant matrices; the parametric uncertainty satisfies

$$
\Delta(t)=[I-F(t) J]^{-1} F(t),
$$

where $J$ is a constant matrix satisfying $I-J J^{T}>0$, and $F(t)$ is an uncertain matrix satisfying $F^{T}(t) F(t) \preceq I$.

Remark 1. The linear fractional parametric uncertainty has been studied in [40-42]; it can be verified that $I-J J^{T}>0$ guarantees that $I-F(t) J$ is invertible for all $F(t)$ satisfying $F^{T}(t) F(t) \preceq I$; the class of parametric uncertainty has been selected because it is very general and includes other classes of uncertainties studied in the literature. Such as when $J=0$, the parametric uncertainty of linear fractional form reduces to norm-bounded parametric uncertainty. So, the results can be easily particularized for this kind of uncertainty.

In fractional differential systems, three kinds of fractional derivatives (i.e., the Grünwald-Letnikov fractional derivative, Riemann-Liouville fractional derivative, and Caputo fractional derivative) have been often used [43-45]; we briefly introduce these three definitions of fractional derivatives as follows. 
Definition 2. The Grünwald-Letnikov fractional derivative with order $\alpha$ of function $x(t)$ is defined below:

$$
{ }_{G L} D_{t, a}^{\alpha} x(t)=\lim _{h \rightarrow 0} \frac{1}{h^{\alpha}} \sum_{j=0}^{[(t-a) / h]}(-1)^{j}\left(\begin{array}{c}
\alpha \\
j
\end{array}\right) x(t-j h),
$$

where

$$
\left(\begin{array}{c}
\alpha \\
j
\end{array}\right)=\frac{\alpha !}{j !(\alpha-j) !}=\frac{\Gamma(\alpha+1)}{\Gamma(j+1) \Gamma(\alpha-j+1)},
$$

and $a$ is a real constant, which expresses a limit value. $[x]$ means the integer part of $x . \Gamma(\cdot)$ is a gamma function given by $\Gamma(z)=\int_{0}^{\infty} t^{z-1} e^{-t} d t$.

Definition 3. The Riemann-Liouville fractional derivative with order $\alpha$ of function $x(t)$ is defined below:

$$
{ }_{R L} D_{t_{0}, t}^{\alpha} x(t)=\frac{1}{\Gamma(m-\alpha)} \frac{d^{m}}{d t^{m}} \int_{t_{0}}^{t}(t-\tau)^{m-\alpha-1} x(\tau) d \tau,
$$

where $t_{0}$ is the initial time, $m-1<\alpha<m \in Z_{+}$.

Definition 4. The Caputo derivative with order $\alpha$ of function $x(t)$ is defined below:

$$
{ }_{C} D_{t_{0}, t}^{\alpha} x(t)=\frac{1}{\Gamma(m-\alpha)} \int_{t_{0}}^{t}(t-\tau)^{m-\alpha-1} x^{(m)}(\tau) d \tau,
$$

where $m-1<\alpha<m \in Z_{+}$.

Remark 5. The Riemann-Liouville derivative and Caputo derivative have been often used in fractional differential systems. But the Laplace transform of Caputo derivative allows utilization of initial values of integer-order derivatives with clear physical interpretations. Moreover, the Caputo definition is more appropriate for describing the initial value problem of fractional differential equations. Therefore, the Caputo derivative definition is adopted in this paper.

Based on output measurement $y_{i}(t)(i=1,2, \ldots, N)$, the following state estimator is constructed as

$$
\begin{array}{r}
{ }_{C} D_{t_{0}, t}^{\alpha} \widehat{x}_{i}(t)=\widehat{A} \widehat{x}_{i}(t)+\sum_{j=1}^{N} G_{i j} \Gamma \widehat{x}_{j}(t)+K\left(y_{i}(t)-\widehat{y}_{i}(t)\right), \\
\widehat{y}_{i}(t)=C \widehat{x}_{i}(t) \quad(i=1,2, \ldots, N),
\end{array}
$$

where $\hat{x}_{i}(t) \in \mathbb{R}^{n}$ is an estimate vector of the network state $x_{i}(t)$, and $\hat{y}_{i}(t) \in \mathbb{R}^{m}$ is an estimate vector of the output $y_{i}(t)$, $K \in R^{n \times m}$ is the estimator matrix to be designed, and $\widehat{A}$ is a unknown system matrix.

Remark 6. As discussed in [36], in addition to estimator matrix $K$, an unknown matrix $\widehat{A}$ is involved in the dynamic of state estimator, which may give a opportunity to better adjust the dynamic characteristics of state estimator.
By using Kronecker product, (1) and (8) can be rewritten in the following compact form:

$$
\begin{aligned}
{ }_{C} D_{t_{0}, t}^{\alpha} x(t) & =\left(I_{N} \otimes A+G \otimes \Gamma\right) x(t), \\
y(t) & =\left(I_{N} \otimes C\right) x(t), \\
{ }_{C} D_{t_{0}, t}^{\alpha} \widehat{x}(t)= & \left(I_{N} \otimes \widehat{A}+G \otimes \Gamma\right) \widehat{x}(t) \\
& +\left(I_{N} \otimes K C\right)(x(t)-\widehat{x}(t)), \\
\hat{y}(t) & =\left(I_{N} \otimes C\right) \hat{x}(t),
\end{aligned}
$$

where

$$
\begin{aligned}
& x(t)=\left[x_{1}^{T}(t), x_{2}^{T}(t), \ldots, x_{N}^{T}(t)\right]^{T}, \\
& y(t)=\left[y_{1}^{T}(t), y_{2}^{T}(t), \ldots, y_{N}^{T}(t)\right]^{T}, \\
& \hat{x}(t)=\left[\hat{x}_{1}^{T}(t), \hat{x}_{2}^{T}(t), \ldots, \hat{x}_{N}^{T}(t)\right]^{T}, \\
& \hat{y}(t)=\left[\hat{y}_{1}^{T}(t), \hat{y}_{2}^{T}(t), \ldots, \hat{y}_{N}^{T}(t)\right]^{T} .
\end{aligned}
$$

Setting $e(t)=x(t)-\hat{x}(t)$, where $e(t)=\left[e_{1}^{T}(t)\right.$, $\left.e_{2}^{T}(t), \ldots, e_{N}^{T}(t)\right]^{T}$, the error dynamics can be obtained from (9) and (10), it follows that

$$
\begin{aligned}
{ }_{C} D_{t_{0}, t}^{\alpha} e(t)= & \left(I_{N} \otimes A-I_{N} \otimes \widehat{A}\right) \widehat{x}(t) \\
& +\left(I_{N} \otimes \widehat{A}+G \otimes \Gamma-I_{N} \otimes K C\right) e(t)
\end{aligned}
$$

Let $X(t)=\left[\hat{x}^{T}(t), e^{T}(t)\right]^{T}$, and the following augmented system can be obtained as follows from (10) and (12):

$$
{ }_{C} D_{t_{0}, t}^{\alpha} X(t)=\mathscr{A} X(t)
$$

where

$$
\mathscr{A}=\left[\begin{array}{cc}
I_{N} \otimes \widehat{A}+G \otimes \Gamma & I_{N} \otimes K C \\
I_{N} \otimes A-I_{N} \otimes \widehat{A} & I_{N} \otimes \widehat{A}+G \otimes \Gamma-I_{N} \otimes K C
\end{array}\right] .
$$

The estimation problem can be transformed to the robust stabilization problem of linear fractional-order system with parametric uncertainty.

Remark 7. As we know, the existing result [46] cannot be applied directly to fractional-order uncertain system (13), as it is hard to compute all eigenvalues of $\mathscr{A}$ in (13); the paper can effectively avoid this difficulty: two unknown matrices $\widehat{A}$ and $K$ can be obtained by using linear matrix inequality technique.

Before giving the main results, the following Lemmas are important and will be used later. 
Lemma 8 (see [47]). Let $a \in \mathbb{R}$, and let $A, B, C$, and $D$ be matrices with appropriate dimensions. By the definition of Kronecker product, the following properties can be proved:

$$
\begin{aligned}
& \text { (1) } a(A \otimes B)=(a A) \otimes B=A \otimes(a B), \\
& \text { (2) }(A \otimes B)^{T}=A^{T} \otimes B^{T}, \\
& \text { (3) }(A \otimes B)(C \otimes D)=(A C) \otimes(B D), \\
& \text { (4) } A \otimes B \otimes C=(A \otimes B) \otimes C=A \otimes(B \otimes C) .
\end{aligned}
$$

Lemma 9 (see [48]). Let $A \in \mathbb{R}^{n \times n}$ be a deterministic real matrix, and then $|\arg (\operatorname{spec}(A))|>\alpha \pi / 2$, where $1 \leq \alpha<2$, if and only if there exists $P>0$ such that

$$
\left[\begin{array}{ll}
\left(A P+P A^{T}\right) \sin \theta & \left(A P-P A^{T}\right) \cos \theta \\
\left(P A^{T}-A P\right) \cos \theta & \left(A P+P A^{T}\right) \sin \theta
\end{array}\right] \prec 0,
$$

where $\theta=\pi-(\alpha \pi / 2)$.

Lemma 10 (see [49]). Suppose that $\Delta(t)$ is given in (2)-(3), with matrices $M=M^{T}, S$, and $N$ of appropriate dimensions, the inequality

$$
M+S \Delta(t) N+N^{T} \Delta^{T}(t) S^{T} \prec 0
$$

holds if and only if for some $\delta>0$

$$
\left[\begin{array}{ccc}
\delta M & S & \delta N^{T} \\
S^{T} & -I & J^{T} \\
\delta N & J & -I
\end{array}\right] \prec 0 .
$$

Similar to [35], for any matrix $\Pi \in \mathbb{R}^{m \times n}$ with full row rank, there exists a singular value decomposition of $\Pi$ as follows:

$$
\Pi=U\left[\begin{array}{ll}
T & 0
\end{array}\right] V^{T},
$$

where $T \in \mathbb{R}^{m \times p}$ is a diagonal matrix with positive elements in decreasing order; $p=\min \{m, n\}, U \in \mathbb{R}^{p \times p}$, and $V \in \mathbb{R}^{n \times n}$ are unitary matrices, then the following lemma holds.

Lemma 11 (see [35]). Given matrix $\Pi \in \mathbb{R}^{m \times n}$ with $\operatorname{rank}(\Pi)=$ $p$, assume that $X \in \mathbb{R}^{n \times n}$ is a symmetric matrix, there exists a matrix $\bar{X} \in \mathbb{R}^{m \times n}$ satisfying $\Pi X=\bar{X} \Pi$ if and only if $X$ can be expressed as

$$
X=V\left[\begin{array}{cc}
X_{11} & 0 \\
0 & X_{22}
\end{array}\right] V^{T},
$$

where $X_{11} \in \mathbb{R}^{m \times m}$ and $X_{22} \in \mathbb{R}^{(n-m) \times(n-m)}$

\section{Main Results}

In this section, firstly, we focus on robust asymptotic stability of uncertain augmented system (13). Secondly, the obtained results will further be extended to design the desired state estimator.
Theorem 12. For given matrices $\widehat{A}$ and $K$, the uncertain fractional-order augmented system (13) with $1 \leq \alpha<2$ is asymptotically stable if there exist positive symmetrical matrices $P_{1}>0, P_{2}>0$ and a positive scalar $\varepsilon>0$ such that the following linear matrix inequality holds:

$$
\left[\begin{array}{ccc}
\operatorname{sym}(\Pi) & * & * \\
\varepsilon S^{T} & -\varepsilon I & * \\
N & \varepsilon J & -\varepsilon I
\end{array}\right] \prec 0,
$$

where

$$
\begin{aligned}
& \operatorname{sym}(\Pi)=\left[\begin{array}{cccc}
\pi_{11} & * & * & * \\
\pi_{21} & \pi_{22} & * & * \\
\pi_{31} & \pi_{32} & \pi_{33} & * \\
\pi_{41} & \pi_{42} & \pi_{43} & \pi_{44}
\end{array}\right], \\
& S=\left[\begin{array}{cccc}
0 & 0 & 0 & 0 \\
\left(I_{N} \otimes D_{A}\right) \sin \theta & 0 & \left(I_{N} \otimes D_{A}\right) \cos \theta & 0 \\
0 & 0 & 0 & 0 \\
-\left(I_{N} \otimes D_{A}\right) \cos \theta & 0 & \left(I_{N} \otimes D_{A}\right) \sin \theta & 0
\end{array}\right], \\
& N=\left[\begin{array}{cccc}
I_{N} \otimes E_{A} P_{1} & I_{N} \otimes E_{A} P_{2} & 0 & 0 \\
0 & 0 & 0 & 0 \\
0 & 0 & I_{N} \otimes E_{A} P_{1} & I_{N} \otimes E_{A} P_{2} \\
0 & 0 & 0 & 0
\end{array}\right], \\
& \pi_{11}=\pi_{33} \\
& =\left(I_{N} \otimes \widehat{A} P_{1}+I_{N} \otimes P_{1} \widehat{A}^{T}+G \otimes \Gamma P_{1}+G^{T} \otimes P_{1} \Gamma^{T}\right) \\
& \times \sin \theta, \\
& \pi_{21}=\pi_{43} \\
& =\left(I_{N} \otimes P_{2} C^{T} K^{T}+I_{N} \otimes A_{0} P_{1}-I_{N} \otimes \widehat{A} P_{1}\right) \sin \theta, \\
& \pi_{22}=\pi_{44} \\
& =\left(I_{N} \otimes A_{0} P_{2}+I_{N} \otimes P_{2} A_{0}^{T}+G \otimes \Gamma P_{2}+G^{T} \otimes P_{2} \Gamma^{T}\right. \\
& \left.-I_{N} \otimes K C P_{2}-I_{N} \otimes P_{2} C^{T} K^{T}\right) \sin \theta, \\
& \pi_{31}=\left(-I_{N} \otimes \widehat{A} P_{1}+I_{N} \otimes P_{1} \widehat{A}^{T}-G \otimes \Gamma P_{1}\right. \\
& \left.+G^{T} \otimes P_{1} \Gamma^{T}\right) \cos \theta, \\
& \pi_{32}=\left(-I_{N} \otimes K C P_{2}+I_{N} \otimes P_{1} A_{0}^{T}-I_{N} \otimes P_{1} \widehat{A}^{T}\right) \cos \theta, \\
& \pi_{41}=\left(I_{N} \otimes P_{2} C^{T} K^{T}-I_{N} \otimes A_{0} P_{1}+I_{N} \otimes \widehat{A} P_{1}\right) \cos \theta, \\
& \pi_{42}=\left(-I_{N} \otimes A_{0} P_{2}+I_{N} \otimes P_{2} A_{0}^{T}-G \otimes \Gamma P_{2}+G^{T} \otimes P_{2} \Gamma^{T}\right. \\
& \left.+I_{N} \otimes K C P_{2}-I_{N} \otimes P_{2} C^{T} K^{T}\right) \cos \theta, \\
& \theta=\pi-\frac{\pi \alpha}{2} \text {. }
\end{aligned}
$$

Proof. If there exist two positive symmetrical matrices $P_{1}$ and $P_{2}$, such that the following matrix inequality holds:

$$
\Phi=\left[\begin{array}{cc}
\left(\mathscr{A} \bar{P}+\bar{P} \mathscr{A}^{T}\right) \sin \theta & \left(\mathscr{A} \bar{P}-\bar{P} \mathscr{A}^{T}\right) \cos \theta \\
\left(-\mathscr{A} \bar{P}+\bar{P} \mathscr{A}^{T}\right) \cos \theta & \left(\mathscr{A} \bar{P}+\bar{P} \mathscr{A}^{T}\right) \sin \theta
\end{array}\right] \prec 0,
$$


where $\bar{P}=\operatorname{diag}\left\{I_{N} \otimes P_{1}, I_{N} \otimes P_{2}\right\}$, then it can be derived from Lemma 9 that uncertain fractional-order augmented system (13) is asymptotically stable.

By some simple computation, we can obtain

$$
\begin{aligned}
& \mathscr{A} \bar{P} \\
& =\left[\begin{array}{cc}
I_{N} \otimes \widehat{A}+G \otimes \Gamma & I_{N} \otimes K C \\
I_{N} \otimes A-I_{N} \otimes \widehat{A} & I_{N} \otimes \widehat{A}+G \otimes \Gamma-I_{N} \otimes K C
\end{array}\right] \\
& \quad \times\left[\begin{array}{cc}
I_{N} \otimes P_{1} & 0 \\
0 & I_{N} \otimes P_{2}
\end{array}\right] \\
& =\left[\begin{array}{cc}
I_{N} \otimes \widehat{A} P_{1}+G \otimes \Gamma P_{1} & I_{N} \otimes K C P_{2} \\
I_{N} \otimes A P_{1}-I_{N} \otimes \widehat{A} P_{1} & I_{N} \otimes \widehat{A} P_{2}+G \otimes \Gamma P_{2}-I_{N} \otimes K C P_{2}
\end{array}\right] .
\end{aligned}
$$

Substituting (24) into (23), it yields

$$
\Phi=\operatorname{sym}(\Pi)+\operatorname{sym}(\Delta),
$$

where

$$
\begin{aligned}
\Pi & =\left[\begin{array}{cccc}
\pi_{1} \sin \theta & \pi_{2} \sin \theta & \pi_{1} \cos \theta & \pi_{2} \cos \theta \\
\pi_{3} \sin \theta & \pi_{4} \sin \theta & \pi_{3} \cos \theta & \pi_{4} \cos \theta \\
-\pi_{1} \cos \theta & -\pi_{2} \cos \theta & \pi_{1} \sin \theta & \pi_{2} \sin \theta \\
-\pi_{3} \cos \theta & -\pi_{4} \cos \theta & \pi_{3} \sin \theta & \pi_{4} \sin \theta
\end{array}\right], \\
\Delta & =\left[\begin{array}{cccc}
0 & 0 & 0 & 0 \\
\Delta_{1} \sin \theta & \Delta_{2} \sin \theta & \Delta_{1} \cos \theta & \Delta_{2} \cos \theta \\
0 & 0 & 0 & 0 \\
-\Delta_{1} \cos \theta & -\Delta_{2} \cos \theta & \Delta_{1} \sin \theta & \Delta_{2} \sin \theta
\end{array}\right], \\
\pi_{1} & =I_{N} \otimes \widehat{A} P_{1}+G \otimes \Gamma P_{1}, \\
\pi_{2} & =I_{N} \otimes K C P_{2}, \\
\pi_{3} & =I_{N} \otimes A_{0} P_{1}-I_{N} \otimes \widehat{A} P_{1}, \\
\pi_{4} & =I_{N} \otimes A_{0} P_{2}+G \otimes \Gamma P_{2}-I_{N} \otimes K C P_{2}, \\
\Delta_{1} & =I_{N} \otimes D_{A} \Delta(t) E_{A} P_{1}, \\
\Delta_{2} & =I_{N} \otimes D_{A} \Delta(t) E_{A} P_{2} .
\end{aligned}
$$

According to (25), one has

$$
\operatorname{sym}(\Delta)=\operatorname{sym}\{S \bar{\Delta}(t) N\}=S \bar{\Delta}(t) N+N^{T} \bar{\Delta}^{T}(t) S^{T},
$$

where

$$
\begin{gathered}
S=\left[\begin{array}{cccc}
0 & 0 & 0 & 0 \\
\left(I_{N} \otimes D_{A}\right) \sin \theta & 0 & \left(I_{N} \otimes D_{A}\right) \cos \theta & 0 \\
0 & 0 & 0 & 0 \\
-\left(I_{N} \otimes D_{A}\right) \cos \theta & 0 & \left(I_{N} \otimes D_{A}\right) \sin \theta & 0
\end{array}\right], \\
N=\left[\begin{array}{cccc}
I_{N} \otimes E_{A} P_{1} & I_{N} \otimes E_{A} P_{2} & 0 & 0 \\
0 & 0 & 0 & 0 \\
0 & 0 & I_{N} \otimes E_{A} P_{1} & I_{N} \otimes E_{A} P_{2} \\
0 & 0 & 0 & 0
\end{array}\right], \\
\bar{\Delta}(t)=\operatorname{diag}\left\{I_{N} \otimes \Delta(t), I_{N} \otimes \Delta(t), I_{N} \otimes \Delta(t), I_{N} \otimes \Delta(t)\right\} .
\end{gathered}
$$

Combining (25) with (27), one has

$$
\Phi=\operatorname{sym}(\Pi)+S \bar{\Delta}(t) N+N^{T} \bar{\Delta}^{T}(t) S^{T} \prec 0 .
$$

By applying Lemma 10, we can obtain that (29) holds, if and only if the following matrix inequality holds

$$
\left[\begin{array}{ccc}
\delta \operatorname{sym}^{T}(\Pi) & * & * \\
S^{T} & -I & * \\
\delta N & J & -I
\end{array}\right] \prec 0,
$$

that is,

$$
\left[\begin{array}{ccc}
\operatorname{sym}(\Pi) & * & * \\
\varepsilon S^{T} & -\varepsilon I & * \\
N & \varepsilon J & -\varepsilon I
\end{array}\right] \prec 0,
$$

where $\varepsilon=1 / \delta$.

The above LMI (31) is just as LMI (21), that is, if (21) holds, it follows from Lemma 9 that uncertain fractionalorder system (13) is asymptotically stable. This completes the proof.

Remark 13. Theorem 12 presents a sufficient condition for asymptotically stable of uncertain fractional-orderaugmented system, which is an LMI condition when matrices $\widehat{A}$ and $K$ are given. If $\widehat{A}$ and $K$ are variables to be determined, owing to the existence of nonlinear terms such as $\widehat{A} P_{1}$ and $K C P_{2}$, the matrix inequality (21) in Theorem 12 is not an LMI, and thus Theorem 12 cannot be used for the estimator's design directly. Our objective hereafter is to provide a design method.

Theorem 14. For the fractional-order complex dynamical networks with linear fractional parametric uncertainty (1), assume that the singular value decomposition of output matrix C with full row rank is

$$
C=U\left[\begin{array}{ll}
T & 0
\end{array}\right] V^{T},
$$

then uncertain fractional-order augmented system (13) with $1 \leq \alpha<2$ is asymptotically stable if there exist symmetrical matrices $P_{1}>0, P_{11}>0, P_{22}>0$ and $X_{1}, X_{2}$ with appropriate dimensions and a scalar $\varepsilon>0$, such that the following linear matrix inequality holds:

$$
\left[\begin{array}{ccc}
\operatorname{sym}(\bar{\Pi}) & * & * \\
\varepsilon S^{T} & -\varepsilon I & * \\
N & \varepsilon J & -\varepsilon I
\end{array}\right]<0,
$$


where

$$
\begin{aligned}
& \operatorname{sym}(\bar{\Pi})=\left[\begin{array}{cccc}
\bar{\pi}_{11} & * & * & * \\
\bar{\pi}_{21} & \bar{\pi}_{22} & * & * \\
\bar{\pi}_{31} & \bar{\pi}_{32} & \bar{\pi}_{33} & * \\
\bar{\pi}_{41} & \bar{\pi}_{42} & \bar{\pi}_{43} & \bar{\pi}_{44}
\end{array}\right], \\
& \bar{\pi}_{11}=\bar{\pi}_{33} \\
& =\left(I_{N} \otimes X_{1}+I_{N} \otimes X_{1}^{T}+G \otimes \Gamma P_{1}+G^{T} \otimes P_{1} \Gamma^{T}\right) \\
& \times \sin \theta \text {, } \\
& \bar{\pi}_{21}=\bar{\pi}_{43} \\
& =\left(I_{N} \otimes C^{T} X_{2}^{T}+I_{N} \otimes A_{0} P_{1}-I_{N} \otimes X_{1}\right) \sin \theta, \\
& \bar{\pi}_{22}=\bar{\pi}_{44} \\
& =\left(I_{N} \otimes A_{0} P_{2}+I_{N} \otimes P_{2} A_{0}^{T}+G \otimes \Gamma P_{2}\right. \\
& \left.+G^{T} \otimes P_{2} \Gamma^{T}-I_{N} \otimes X_{2} C-I_{N} \otimes C^{T} X_{2}^{T}\right) \\
& \times \sin \theta, \\
& \bar{\pi}_{31}=\left(-I_{N} \otimes X_{1}+I_{N} \otimes X_{1}^{T}-G \otimes \Gamma P_{1}+G^{T} \otimes P_{1} \Gamma^{T}\right) \\
& \times \cos \theta \\
& \bar{\pi}_{32}=\left(-I_{N} \otimes X_{2} C+I_{N} \otimes P_{1} A_{0}^{T}-I_{N} \otimes X_{1}^{T}\right) \cos \theta, \\
& \bar{\pi}_{41}=\left(I_{N} \otimes C^{T} X_{2}^{T}-I_{N} \otimes A_{0} P_{1}+I_{N} \otimes X_{1}\right) \cos \theta, \\
& \bar{\pi}_{42}=\left(-I_{N} \otimes A_{0} P_{2}+I_{N} \otimes P_{2} A_{0}^{T}-G \otimes \Gamma P_{2}\right. \\
& \left.+G^{T} \otimes P_{2} \Gamma^{T}+I_{N} \otimes X_{2} C-I_{N} \otimes C^{T} X_{2}^{T}\right) \cos \theta, \\
& \theta=\pi-\frac{\pi \alpha}{2} \\
& P_{2}=V\left[\begin{array}{cc}
P_{11} & 0 \\
0 & P_{22}
\end{array}\right] V^{T} \text {. }
\end{aligned}
$$

Moreover, two unknown estimator gain matrices are given as

$$
\widehat{A}=X_{1} P_{1}^{-1}, \quad K=X_{2} U T P_{11}^{-1} T^{-1} U^{-1} .
$$

Proof. Since

$$
C=U\left[\begin{array}{ll}
T & 0
\end{array}\right] V^{T}, \quad P_{2}=V\left[\begin{array}{cc}
P_{11} & 0 \\
0 & P_{22}
\end{array}\right] V^{T} .
$$

From Lemma 11, there exists $\bar{P}_{2}=U T P_{11} T^{-1} U^{-1}$, such that $C P_{2}=\bar{P}_{2} C$; it is easily obtained that $\bar{P}_{2}^{-1}=U T P_{11}^{-1} T^{-1} U^{-1}$. Setting $X_{1}=\widehat{A} P_{1}$ and $X_{2}=K \bar{P}_{2}$, then matrix inequality (21) is equivalent to (33). Moreover, estimator gain matrices can be obtained as follows:

$$
\begin{gathered}
\widehat{A}=X_{1} P_{1}^{-1}, \\
K=X_{2} \bar{P}_{2}^{-1}=X_{2} U T P_{11}^{-1} T^{-1} U^{-1} .
\end{gathered}
$$

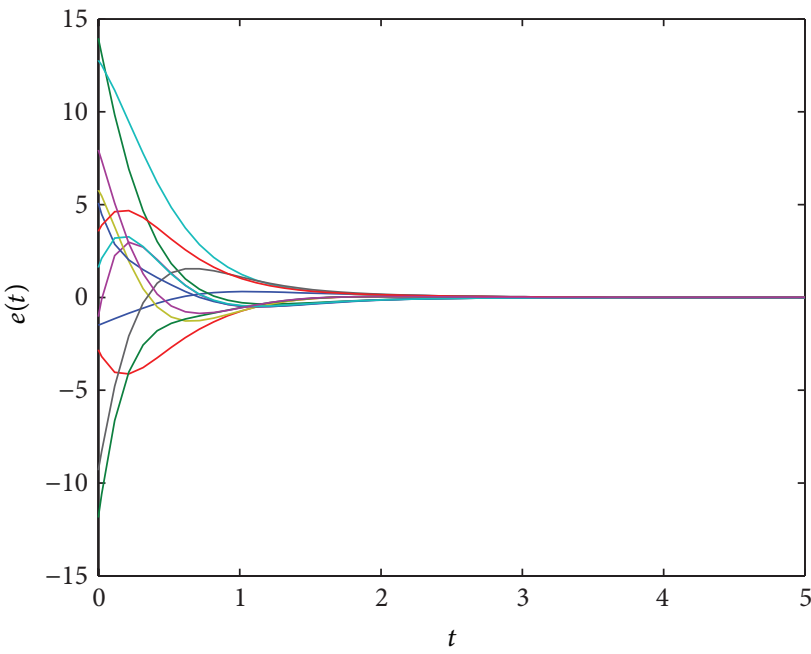

Figure 1: The curve of error $e(t)$ between the state $x(t)$ and its estimate $\widehat{x}(t)$.

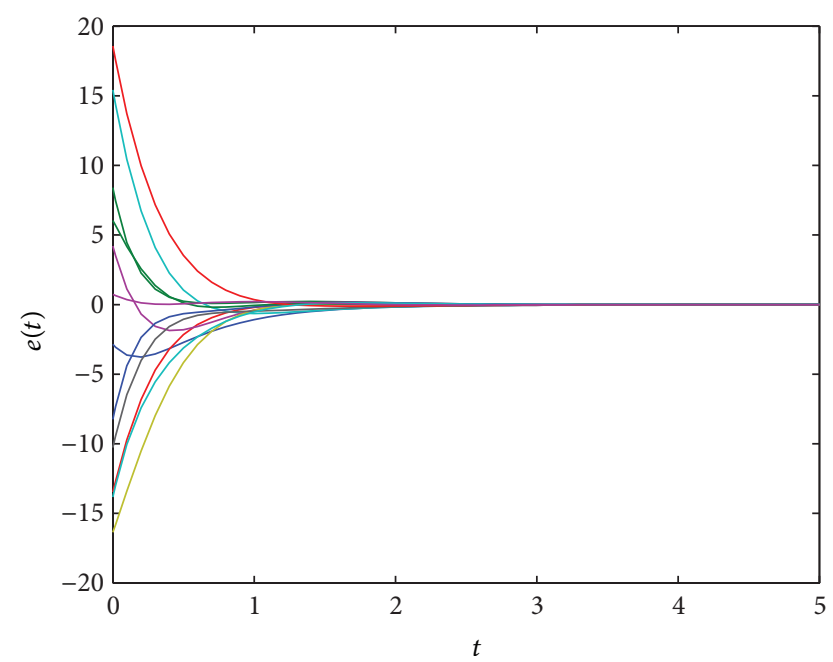

Figure 2: The curve of error $e(t)$ between the state $x(t)$ and its estimate $\widehat{x}(t)$.

In particular, setting $\widehat{A}=A_{0}$ in (8), then the state estimator will reduce to

$$
\begin{array}{r}
{ }_{C} D_{t_{0}, t}^{\alpha} \widehat{x}_{i}(t)=A_{0} \widehat{x}_{i}(t)+\sum_{j=1}^{N} G_{i j} \Gamma \widehat{x}_{j}(t)+K\left(y_{i}(t)-\widehat{y}_{i}(t)\right), \\
\widehat{y}_{i}(t)=C \hat{x}_{i}(t) \quad(i=1,2, \ldots, N) .
\end{array}
$$

The corresponding augmented system is described as

$$
{ }_{C} D_{t_{0}, t}^{\alpha} X(t)=\overline{\mathscr{A}} X(t)
$$

where

$$
\overline{\mathscr{A}}=\left[\begin{array}{cc}
I_{N} \otimes A_{0}+G \otimes \Gamma & I_{N} \otimes K C \\
I_{N} \otimes A-I_{N} \otimes A_{0} & I_{N} \otimes \widehat{A}+G \otimes \Gamma-I_{N} \otimes K C
\end{array}\right] .
$$


TABLE 1: Estimator matrices $\widehat{A}$ and $K$ for different fractional orders $\alpha$.

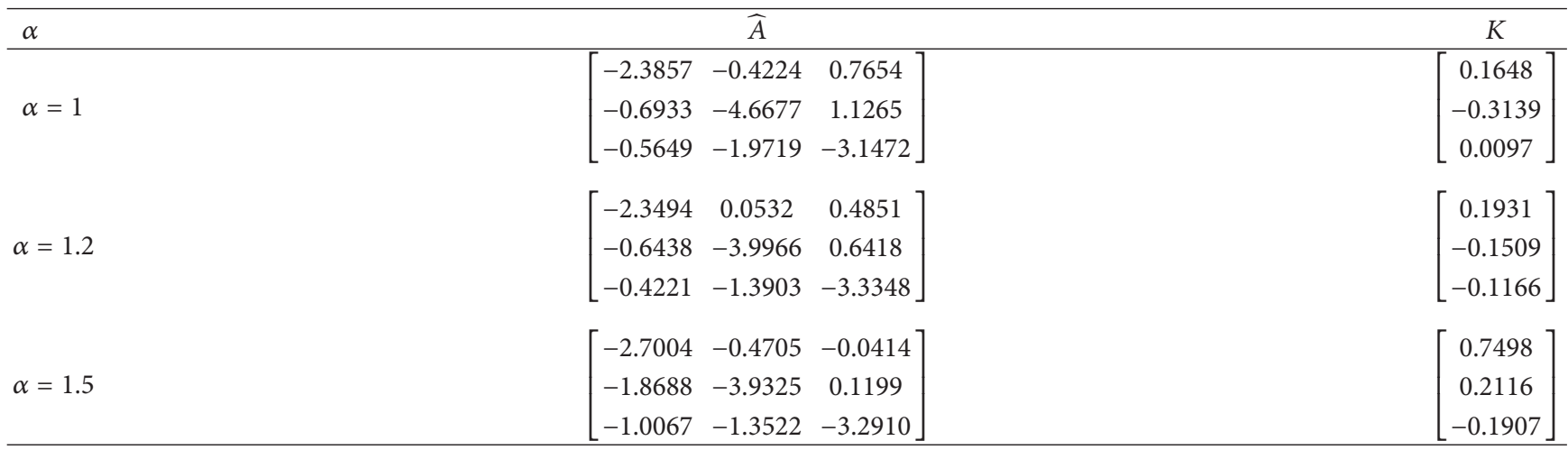

TABLE 2: Estimator matrix $K$ for different fractional orders $\alpha$.

\begin{tabular}{lccc}
\hline$\alpha$ & 1 & 1.2 & 1.5 \\
\hline$K$ & {$[0.1349,-0.0787,0.0484]^{T}$} & {$[0.1858,-0.0462,-0.0023]^{T}$} & {$[0.4464,-0.0117,-0.2841]^{T}$} \\
\hline
\end{tabular}

The unknown estimator matrix $K$ can be solved from the following corollary.

Corollary 15. For the fractional-order complex dynamical networks with linear fractional parametric uncertainty (1), assume that the singular value decomposition of output matrix $C$ with full row rank is

$$
C=U\left[\begin{array}{ll}
T & 0
\end{array}\right] V^{T},
$$

then uncertain fractional-order augmented system (39) with $1 \leq \alpha<2$ is asymptotically stable if there exist symmetrical matrices $P_{1}>0, P_{11}>0, P_{22}>0$ and $X_{1}$ with appropriate dimensions and a scalar $\varepsilon>0$, such that the following linear matrix inequality holds:

$$
\left[\begin{array}{ccc}
\operatorname{sym}(\widetilde{\Pi}) & * & * \\
\varepsilon S^{T} & -\varepsilon I & * \\
N & \varepsilon J & -\varepsilon I
\end{array}\right] \prec 0,
$$

where

$$
\begin{gathered}
\operatorname{sym}(\widetilde{\Pi})=\left[\begin{array}{cccc}
\tilde{\pi}_{11} & * & * & * \\
\tilde{\pi}_{21} & \bar{\pi}_{22} & * & * \\
\tilde{\pi}_{31} & \tilde{\pi}_{32} & \tilde{\pi}_{33} & * \\
\tilde{\pi}_{41} & \bar{\pi}_{42} & \tilde{\pi}_{43} & \bar{\pi}_{44}
\end{array}\right], \\
\tilde{\pi}_{11}=\tilde{\pi}_{33}=\left(I_{N} \otimes A_{0} P_{1}+I_{N} \otimes P_{1} A_{0}^{T}+G \otimes \Gamma P_{1}\right. \\
\left.+G^{T} \otimes P_{1} \Gamma^{T}\right) \sin \theta, \\
\tilde{\pi}_{21}=\tilde{\pi}_{43}=\left(I_{N} \otimes C^{T} X_{1}^{T}\right) \sin \theta,
\end{gathered}
$$

$$
\begin{aligned}
& \tilde{\pi}_{31}=\left(-I_{N} \otimes A_{0} P_{1}+I_{N} \otimes P_{1} A_{0}^{T}-G \otimes \Gamma P_{1}\right. \\
&\left.+G^{T} \otimes P_{1} \Gamma^{T}\right) \cos \theta, \\
& \tilde{\pi}_{32}=\left(-I_{N} \otimes X_{1} C\right) \cos \theta, \\
& \tilde{\pi}_{41}=\left(I_{N} \otimes C^{T} X_{1}^{T}\right) \cos \theta, \\
& P_{2}=V\left[\begin{array}{cc}
P_{11} & 0 \\
0 & P_{22}
\end{array}\right] V^{T} .
\end{aligned}
$$

The desired estimator gain matrix is given as

$$
K=X_{1} U T P_{11}^{-1} T^{-1} U^{-1}
$$

Remark 16. The state estimation discussed in this paper is fairly comprehensive; our results can readily specialize to many special cases, such as, when $J=0$, it implies that $\Delta(t)=$ $F(t)$ with $F^{T}(t) F(t) \preceq I$; that is, the parametric uncertainty of linear fractional form reduces to norm-bounded parametric uncertainty; the corresponding result can be easily derived from Theorem 14. Let $\widehat{A}=A_{0}$ and $J=0$ in (2) and (8), respectively, the problem will reduce state estimation for a class of fractional-order complex dynamical networks with norm-bounded parametric uncertainty; the corresponding result can be obtained from Corollary 15 . The specialized results are still believed to be new and have not been fully researched yet. For presentation, we omit the corresponding corollaries here.

\section{Numerical Examples}

In this section, two different fractional-order systems are given as examples to verify the effectiveness of the control scheme described in the preceding section. 
Example 17. Consider the fractional-order complex dynamical networks (1) and the state estimator (8), the relevant parameters are given as follows:

$$
\begin{gathered}
A_{0}=\left[\begin{array}{ccc}
-1.5 & 0.5 & 1 \\
-1 & -3 & 1 \\
-0.5 & -1.5 & -2.5
\end{array}\right], \quad C=\left[\begin{array}{c}
1.5 \\
2 \\
1
\end{array}\right], \\
G=\left[\begin{array}{cccc}
-1 & 0 & 1 & 0 \\
1 & -1 & 0 & 0 \\
0 & 1 & -1 & 0 \\
1 & 0 & 0 & -1
\end{array}\right], \quad \Gamma=\left[\begin{array}{lll}
1 & 0 & 0 \\
0 & 1 & 0 \\
0 & 0 & 1
\end{array}\right], \\
D_{A}=\left[\begin{array}{ccc}
-0.1 & 0.05 & 0.1 \\
-0.1 & -0.23 & 0.1 \\
-0.15 & -0.2 & -0.14
\end{array}\right], \quad E_{A}=\left[\begin{array}{lll}
1 & 0 & 0 \\
0 & 1 & 0 \\
0 & 0 & 1
\end{array}\right], \\
F(t)=\left[\begin{array}{ccc}
\sin (0.1 \pi t) & 0 \\
0 & \cos (0.1 \pi t) \\
0 & 0 & \sin (0.1 \pi t)
\end{array}\right], \\
J=\left[\begin{array}{ccc}
0.5 & 0 & 0 \\
0 & 0.5 & 0 \\
0 & 0 & 0.5
\end{array}\right] .
\end{gathered}
$$

It is easy to verify that $C=[1.5,2,1]$ is full row rank; by using matrix's singular value decomposition, we can obtain

$$
U=1, \quad T=1.5, \quad V=\left[\begin{array}{ccc}
1 & 0 & 0 \\
\frac{4}{3} & 1 & 0 \\
\frac{2}{3} & 0 & 1
\end{array}\right] .
$$

In Theorem 14, setting fractional-order $\alpha=1$, a set of feasible solutions can be obtained using the Matlab Control Toolbox as follows:

$$
\begin{gathered}
X_{1}=\left[\begin{array}{ccc}
-2.9462 & 0.0227 & 0.7604 \\
-0.1659 & -3.4440 & 1.2318 \\
-0.4875 & -1.3645 & -3.2833
\end{array}\right], \\
X_{2}=\left[\begin{array}{c}
0.2473 \\
-0.4712 \\
0.0146
\end{array}\right], \\
P_{1}=\left[\begin{array}{ccc}
1.2675 & -0.1479 & 0.0201 \\
-0.1479 & 0.7565 & -0.0139 \\
0.0201 & -0.0139 & 1.0483
\end{array}\right], \\
P_{11}=1.5008, \quad P_{22}=\left[\begin{array}{cc}
1.3876 & -0.0264 \\
-0.0264 & 1.2900
\end{array}\right] ;
\end{gathered}
$$

then two unknown estimator gain matrices are given as

$$
\begin{gathered}
\widehat{A}=X_{1} P_{1}^{-1}=\left[\begin{array}{ccc}
-2.3875 & -0.4224 & 0.7654 \\
-0.6933 & -4.6677 & 1.1265 \\
-0.5649 & -1.9719 & -3.1472
\end{array}\right], \\
K=X_{2} U T P_{11}^{-1} T^{-1} U^{-1}=\left[\begin{array}{c}
0.1648 \\
-0.3139 \\
0.0097
\end{array}\right] .
\end{gathered}
$$

Therefore, it follows from Theorem 14 that uncertain fractional-order augmented system (13) is asymptotically stable. The response of error dynamics converges to zero asymptotically, which is given in Figure 1; it can be seen that the simulation has confirmed that the designed estimators perform very well. Two unknown estimator gain matrices are listed in Table 1 for different fractional orders.

Example 18. If $\widehat{A}=A_{0}$ in estimator (8), the relevant parameters are given in Example 17. Setting fractional-order $\alpha=1$, using the Matlab LMI toolbox to solve the LMI in Corollary 15, we can obtain the following matrices:

$$
\begin{gathered}
X_{1}=\left[\begin{array}{c}
0.1920 \\
-0.1120 \\
0.0689
\end{array}\right], \\
P_{1}=\left[\begin{array}{ccc}
1.6859 & -0.1948 & 0.0626 \\
-0.1948 & 1.1260 & -0.0432 \\
0.0626 & -0.0432 & 1.2487
\end{array}\right], \\
P_{11}=1.4233, \quad P_{22}=\left[\begin{array}{cc}
1.1486 & -0.0333 \\
-0.0333 & 1.2540
\end{array}\right] ;
\end{gathered}
$$

then the estimator gain matrix is given as

$$
\begin{aligned}
K & =X_{1} U T P_{11}^{-1} T^{-1} U^{-1} \\
& =\left[\begin{array}{lll}
0.1349 & -0.0787 & 0.0484
\end{array}\right]^{T} .
\end{aligned}
$$

It is not difficult to verify that, with the obtained estimator gain $K$, the response of error dynamics converges to zero asymptotically; the corresponding simulation result is shown in Figure 2. The estimator gain matrix is given in Table 2 for different fractional orders.

\section{Conclusions}

State estimation problem is investigated for a class of fractional-order complex dynamical networks with parametric uncertainty. By using matrix's singular value decomposition and LMI techniques, the existence conditions of designing state estimator derived are in the form of linear matrix inequalities which can be readily solved using the LMI toolbox. Finally, two numerical examples are provided to demonstrate the validity of this approach.

\section{Acknowledgments}

This work was jointly supported by the National Science Foundation of China (Grant nos. 61272034, 61074024, and 60874113), the Natural Science Foundation of Jiangsu Province of China (Grant no. BK2010543), the Education Department Research Project of Zhejiang Province of China (Grant no. Y201019013), and the Outstanding Young Teacher Project of Zhejiang Province of China.

\section{References}

[1] M. E. Crovella and A. Bestavros, "Self-similarity in world wide web traffic: evidence and possible causes," IEEE/ACM Transactions on Networking, vol. 5, no. 6, pp. 835-846, 1997. 
[2] J. W. Moon and Y. G. Kim, "Extending the TAM for a worldwide-web context," Information and Management, vol. 38, no. 4, pp. 217-230, 2001.

[3] Z. Wang, Y. Liu, and X. Liu, "State estimation for jumping recurrent neural networks with discrete and distributed delays," Neural Networks, vol. 22, no. 1, pp. 41-48, 2009.

[4] J. Cao and L. Li, "Cluster synchronization in an array of hybrid coupled neural networks with delay," Neural Networks, vol. 22, no. 4, pp. 335-342, 2009.

[5] G. Robins, P. Pattison, and P. Wang, "Closure, connectivity and degree distributions: exponential random graph $\left(\mathrm{p}^{*}\right)$ models for directed social networks," Social Networks, vol. 31, no. 2, pp. 105117, 2009.

[6] A. Clifton, E. Turkheimer, and T. F. Oltmanns, "Personality disorder in social networks: Network position as a marker of interpersonal dysfunction," Social Networks, vol. 31, no. 1, pp. 26-32, 2009.

[7] Z. Han, T. Himsoon, W. P. Siriwongpairat, and K. J. R. Liu, "Resource allocation for multiuser cooperative OFDM networks: who helps whom and how to cooperate," IEEE Transactions on Vehicular Technology, vol. 58, no. 5, pp. 23782391, 2009.

[8] R. Canipari, V. Cellini, and S. Cecconi, "The ovary feels fine when paracrine and autocrine networks cooperate with gonadotropins in the regulation of folliculogenesis," Current Pharmaceutical Design, vol. 18, no. 3, pp. 245-255, 2012.

[9] L. Jarosz, "The city in the country: growing alternative food networks in Metropolitan areas," Journal of Rural Studies, vol. 24, no. 3, pp. 231-244, 2008.

[10] S. Lockie, "Responsibility and agency within alternative food networks: assembling the 'citizen consumer," Agriculture and Human Values, vol. 26, no. 3, pp. 193-201, 2009.

[11] F. B. Amar and R. Dhifaoui, "Study of the periodic ferroresonance in the electrical power networks by bifurcation diagrams," International Journal of Electrical Power and Energy Systems, vol. 33, no. 1, pp. 61-85, 2011.

[12] C. Dangalchev, "Generation models for scale-free networks," Physica A, vol. 338, no. 3-4, pp. 659-671, 2004.

[13] X. Wang and G. Chen, "Synchronization in complex dynamical networks," Journal of Systems Science and Complexity, vol. 16, no. 3, pp. 358-371, 2003.

[14] X. Li, X. Wang, and G. Chen, "Pinning a complex dynamical network to its equilibrium," IEEE Transactions on Circuits and Systems I, vol. 51, no. 10, pp. 2074-2087, 2004.

[15] Z. Li and G. Chen, "Global synchronization and asymptotic stability of complex dynamical networks," IEEE Transactions on Circuits and Systems II, vol. 53, no. 1, pp. 28-33, 2006.

[16] J. Liang, Z. Wang, and X. Liu, "Exponential synchronization of stochastic delayed discrete-time complex networks," Nonlinear Dynamics, vol. 53, no. 1-2, pp. 153-165, 2008.

[17] H. Gao, J. Lam, and G. Chen, "New criteria for synchronization stability of general complex dynamical networks with coupling delays," Physics Letters A, vol. 360, no. 2, pp. 263-273, 2006.

[18] X. Liu and T. Chen, "Exponential synchronization of nonlinear coupled dynamical networks with a delayed coupling," Physica A, vol. 381, no. 1-2, pp. 82-92, 2007.

[19] J. Cao and J. Lu, "Adaptive synchronization of neural networks with or without time-varying delay," Chaos, vol. 16, no. 1, Article ID 013133, 6 pages, 2006.

[20] Z. Duan, G. Chen, and L. Huang, "Synchronization of weighted networks and complex synchronized regions," Physics Letters A, vol. 372, no. 21, pp. 3741-3751, 2008.
[21] Z. Wang, D. W. C. Ho, and X. Liu, "State estimation for delayed neural networks," IEEE Transactions on Neural Networks, vol. 16, no. 1, pp. 279-284, 2005.

[22] Y. Liu, Z. Wang, J. Liang, and X. Liu, "Synchronization and state estimation for discrete-time complex networks with distributed delays," IEEE Transactions on Systems, Man, and Cybernetics B, vol. 38, no. 5, pp. 1314-1325, 2008.

[23] J. Liang, Z. Wang, and X. Liu, "State estimation for coupled uncertain stochastic networks with missing measurements and time-varying delays: the discrete-time case," IEEE Transactions on Neural Networks, vol. 20, no. 5, pp. 781-793, 2009.

[24] J. Liang, Z. Wang, and X. Liu, "Distributed state estimation for discrete-time sensor networks with randomly varying nonlinearities and missing measurements," IEEE Transactions on Neural Networks, vol. 22, no. 3, pp. 486-496, 2011.

[25] B. Shen, Z. Wang, and X. Liu, "Bounded $H_{\infty}$ synchronization and state estimation for discrete time-varying stochastic complex networks over a finite horizon," IEEE Transactions on Neural Networks, vol. 22, no. 1, pp. 145-157, 2011.

[26] P. Balasubramaniam, S. Lakshmanan, and S. J. S. Theesar, "State estimation for Markovian jumping recurrent neural networks with interval time-varying delays," Nonlinear Dynamics, vol. 60, no. 4, pp. 661-675, 2010.

[27] P. Balasubramaniam, V. Vembarasan, and R. Rakkiyappan, "Delay-dependent robust exponential state estimation of Markovian jumping fuzzy Hopfield neural networks with mixed random time-varying delays," Communications in Nonlinear Science and Numerical Simulation, vol. 16, no. 4, pp. 2109-2129, 2011.

[28] P. Balasubramaniam, V. Vembarasan, and R. Rakkiyappan, "Delay-dependent robust asymptotic state estimation of TakagiSugeno fuzzy Hopfield neural networks with mixed interval time-varying delays," Expert Systems with Applications, vol. 39, no. 1, pp. 472-481, 2012.

[29] R. Bagley and P. Torvik, "On the fractional calculus model of viscoelastic behavior," Journal of Rheology, vol. 30, no. 1, pp. 133155, 1986.

[30] A. A. Stanislavsky, "The peculiarity of self-excited oscillations in fractional systems," Acta Physica Polonica B, vol. 37, no. 2, pp. 319-329, 2006.

[31] V. E. Tarasov and M. Edelman, "Fractional dissipative standard map," Chaos, vol. 20, no. 2, Article ID 023127, 7 pages, 2010.

[32] C. P. Li and Z. G. Zhao, "Introduction to fractional integrability and differentiability," The European Physical Journal-Special Topics, vol. 193, no. 1, pp. 5-26, 2011.

[33] J. G. Lu and G. Chen, "Robust stability and stabilization of fractional-order interval systems: an LMI approach," IEEE Transactions on Automatic Control, vol. 54, no. 6, pp. 1294-1299, 2009.

[34] J. G. Lu and Y. Q. Chen, "Robust stability and stabilization of fractional-order interval systems with the fractional order $\alpha$ : the $0 \ll \alpha \ll 1$ case," IEEE Transactions on Automatic Control, vol. 55, no. 1, pp. 152-158, 2010.

[35] Y.-H. Lan and Y. Zhou, "LMI-based robust control of fractionalorder uncertain linear systems," Computers and Mathematics with Applications, vol. 62, no. 3, pp. 1460-1471, 2011.

[36] Y. H. Lan, H. X. Huang, and Y. Zhou, "Observer-based robust control of $a(1 \leq a<2)$ fractional-order uncertain systems: a linear matrix inequality approach," IET Control Theory and Applications, vol. 6, no. 2, pp. 229-234, 2012. 
[37] Y. Tang, Z. Wang, and J. A. Fang, "Pinning control of fractionalorder weighted complex networks," Chaos, vol. 19, no. 1, Article ID 013112, 9 pages, 2009.

[38] X. J. Wu and H. T. Lu, "Outer synchronization between two differentfractional-order general complex dynamical networks," Chinese Physics B, vol. 19, no. 7, Article ID 070511, 12 pages, 2009.

[39] W. K. Wong, H. Li, and S. Y. S. Leung, "Robust synchronization of fractional-order complex dynamical networks with parametric uncertainties," Communications in Nonlinear Science and Numerical Simulation, vol. 17, no. 12, pp. 4877-4890, 2012.

[40] L. Guo, " $H_{\infty}$ output feedback control for delay systems with nonlinear and parametric uncertainties," IEE Proceedings Control Theory and Applications, vol. 149, no. 3, pp. 226-236, 2002.

[41] W. Qian, T. Li, S. Cong, and S. Fei, "Improved stability analysis on delayed neural networks with linear fractional uncertainties," Applied Mathematics and Computation, vol. 217, no. 7, pp. 3596-3606, 2010.

[42] X. Li, H. Gao, and X. Yu, "A unified approach to the stability of generalized static neural networks with linear fractional uncertainties and delays," IEEE Transactions on Systems, Man, and Cybernetics B, vol. 41, no. 5, pp. 1275-1286, 2011.

[43] K. B. Oldham and J. Spanier, The Fractional Calculus, Elsevier Science, Amsterdam, The Netherlands, 1974.

[44] J. Sabatier, O. P. Agrawal, and J. A. Machado, Advances in Fractional Calculus: Theoretical Developments and Applications in Physics and Engineering, Springer, New York, NY, USA, 2007.

[45] C. P. Li and F. R. Zhang, "A survey on the stability of fractional differential equations," The European Physical Journal-Special Topics, vol. 193, no. 1, pp. 27-47, 2011.

[46] M. Moze, J. Sabatier, and A. Oustaloup, "LMI tools for stability analysis of fractional systems," in Proceedings of the ASME International Design Engineering Technical Conferences and Computers and Information in Engineering Conference (IDETC/CIE'05), pp. 1611-1619, Long Beach, Calif, USA, September 2005.

[47] H. Li, "Cluster synchronization stability for stochastic complex dynamical networks with probabilistic interval time-varying delays," Journal of Physics A, vol. 44, no. 10, Article ID 105101, 24 pages, 2011.

[48] M. Chilali, P. Gahinet, and P. Apkarian, "Robust pole placement in LMI regions," IEEE Transactions on Automatic Control, vol. 44, no. 12, pp. 2257-2270, 1999.

[49] S. Zhou and J. Lam, "Robust stabilization of delayed singular systems with linear fractional parametric uncertainties," Circuits, Systems, and Signal Processing, vol. 22, no. 6, pp. 579-588, 2003. 


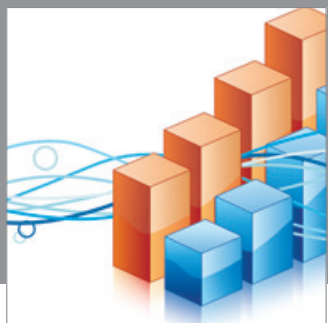

Advances in

Operations Research

mansans

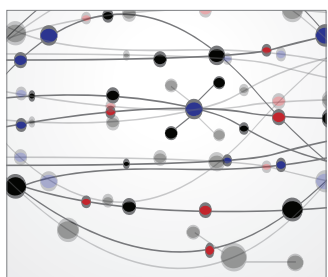

The Scientific World Journal
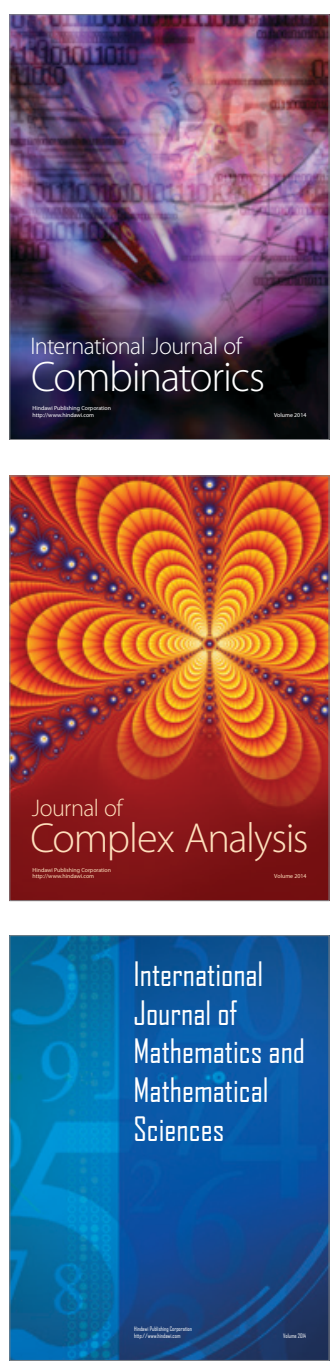
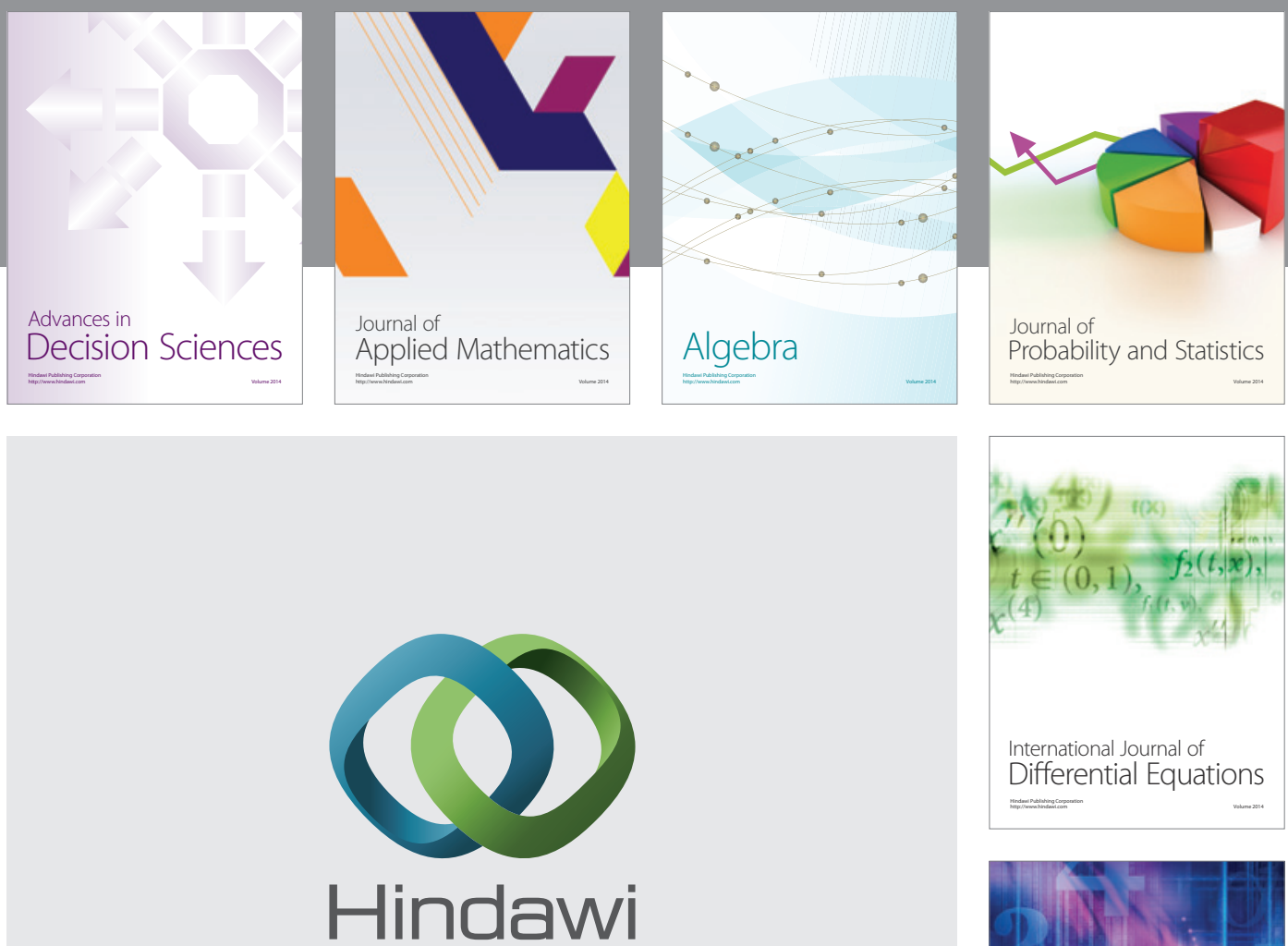

Submit your manuscripts at http://www.hindawi.com
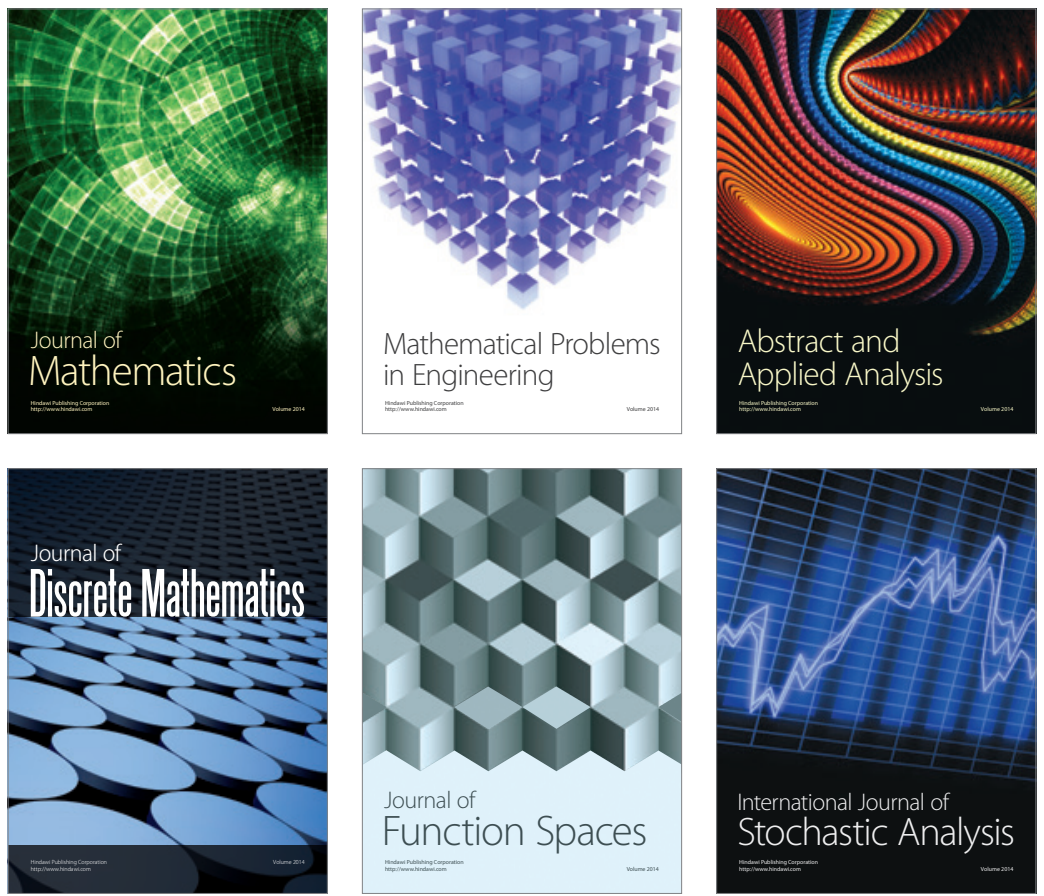

Journal of

Function Spaces

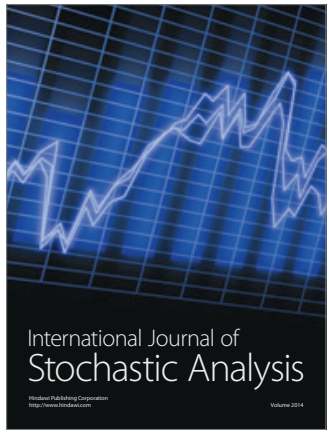

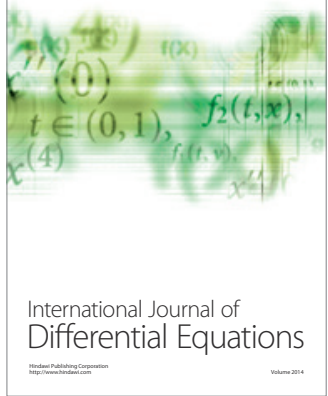
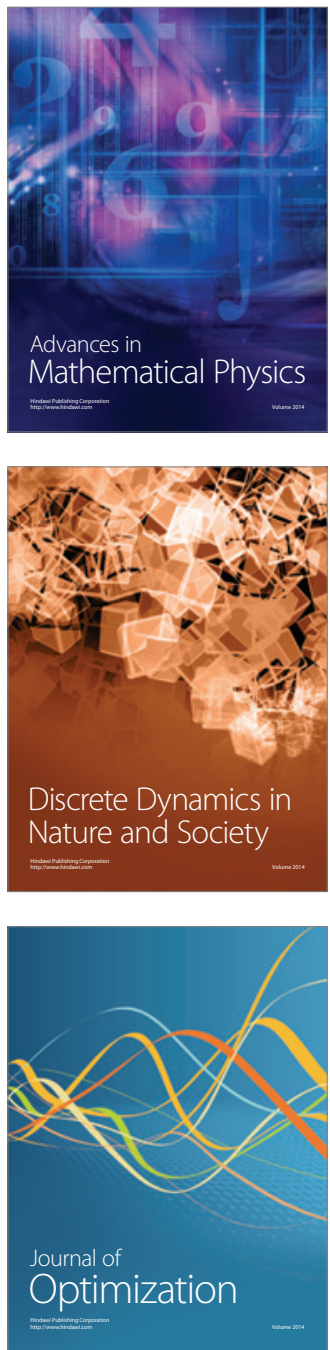